Acta Crystallographica Section B

Structural

Science

ISSN 0108-7681

Editor: Carolyn P. Brock

\title{
Structure of tris(hydroxymethyl)acetic acid
}

D. Eilerman and R. Rudman

This electronic document was scanned from an archival copy of material deposited to accompany a paper published in an IUCr journal. In many cases the only accessible copy was a microfilm of a poor-quality original. 


\section{$34588 *$}

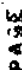

\section{空}

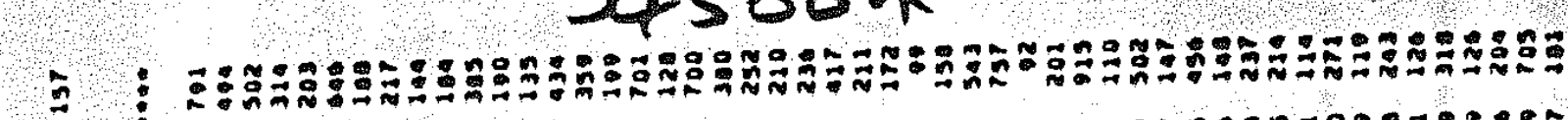

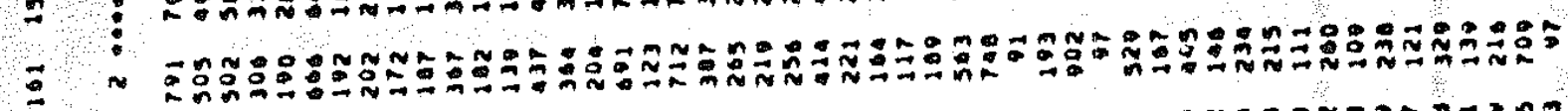
?

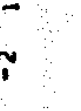

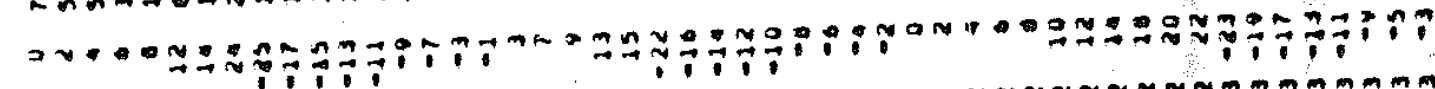

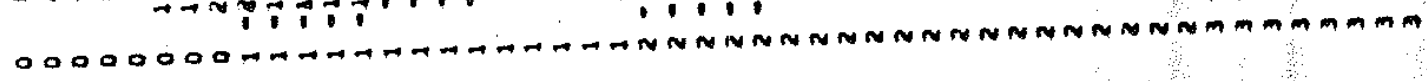
$\rightarrow$

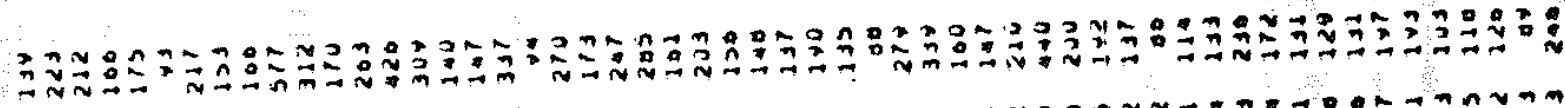

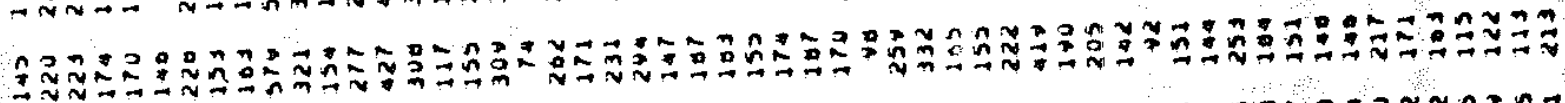

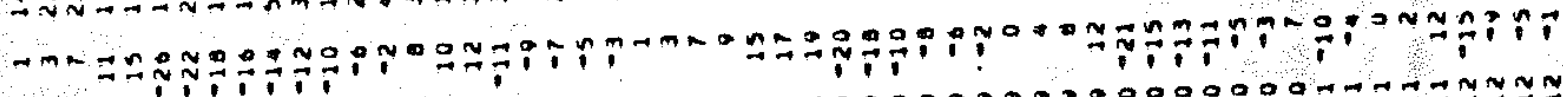

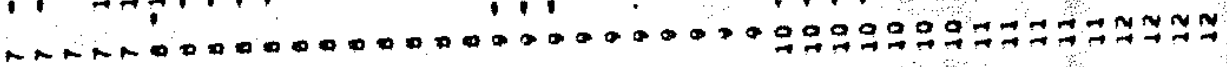

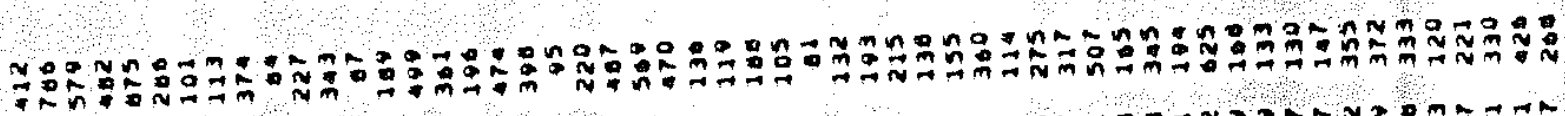

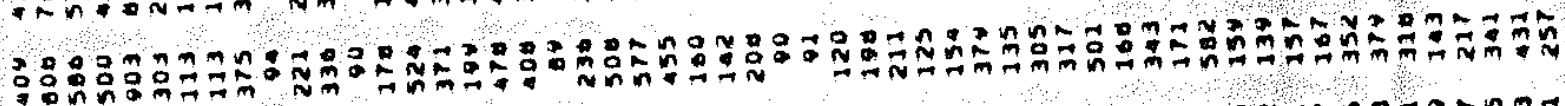

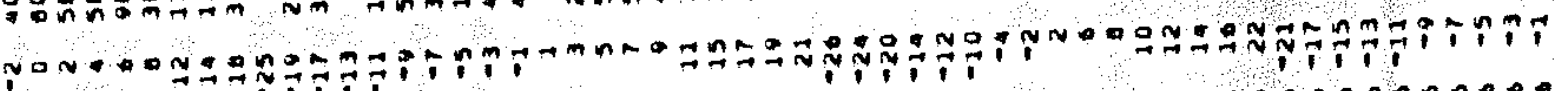

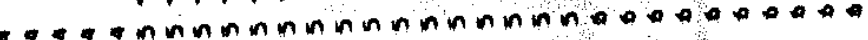
$m m m m m m m m-1+\cdots+\infty$

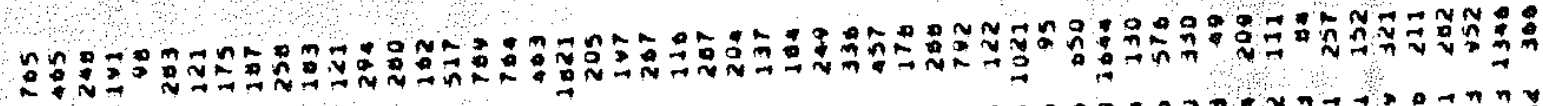

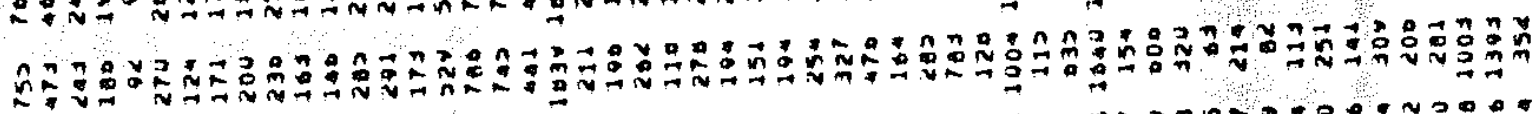

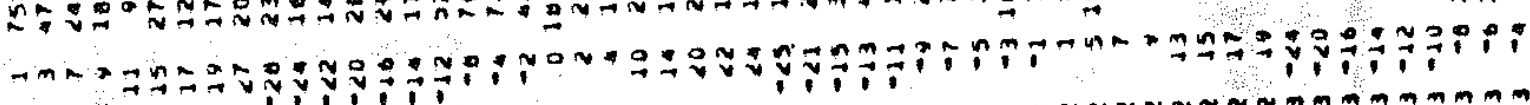

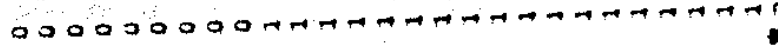

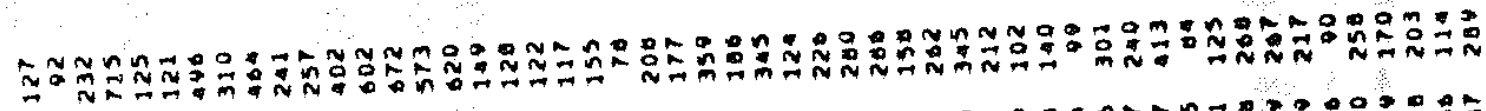

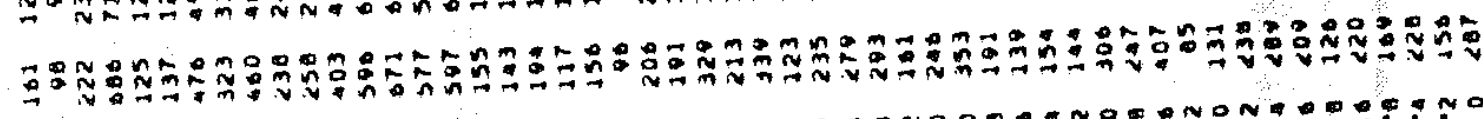

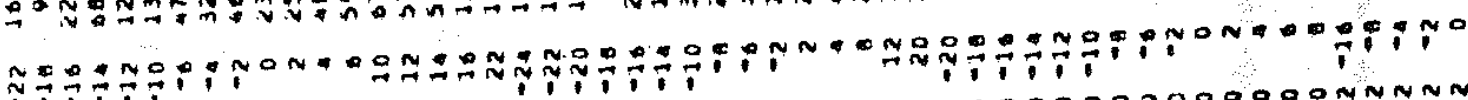
1

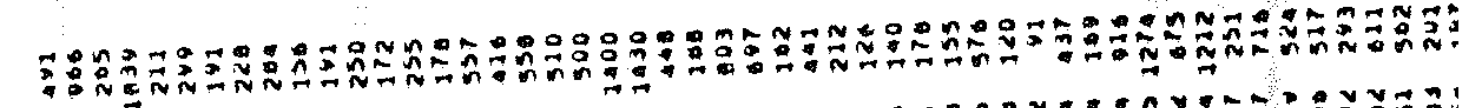

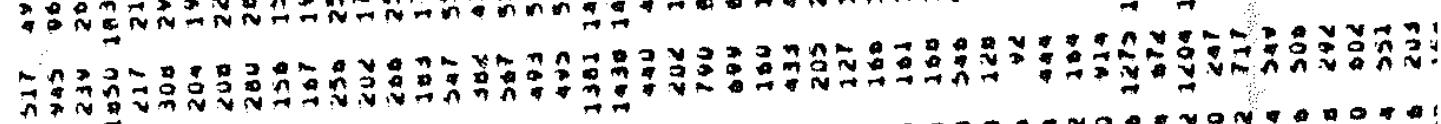

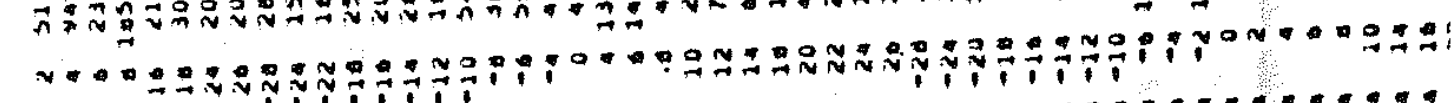

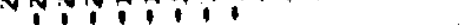

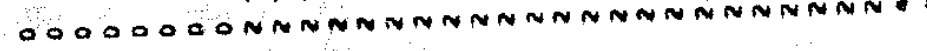




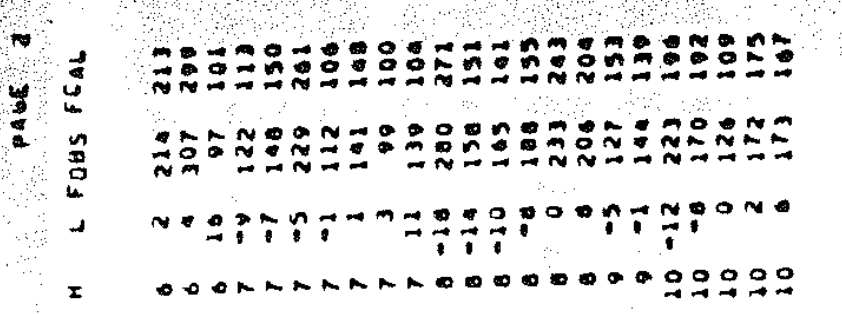

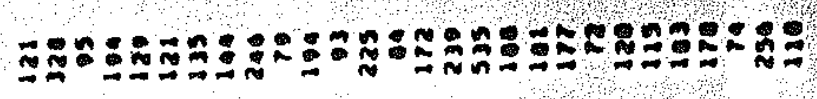

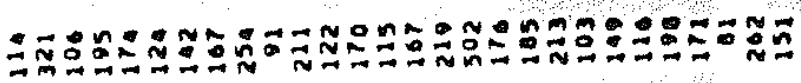 ma 000000-m-m-m - ANNNNNNNNNmmmmmm

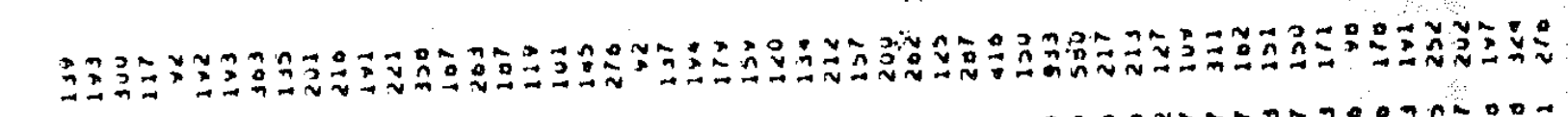

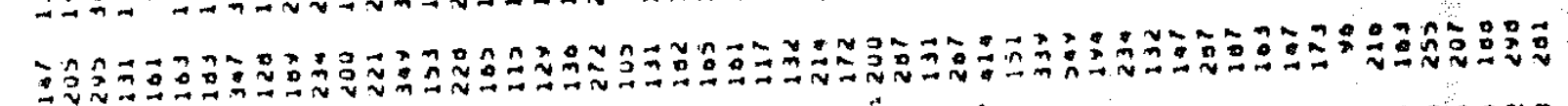

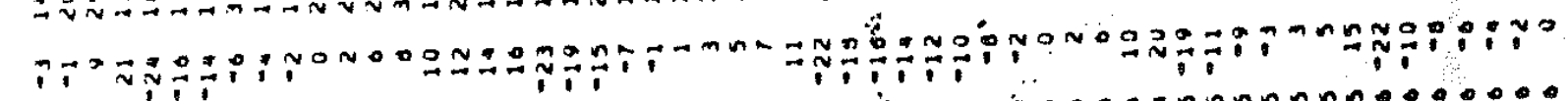
-

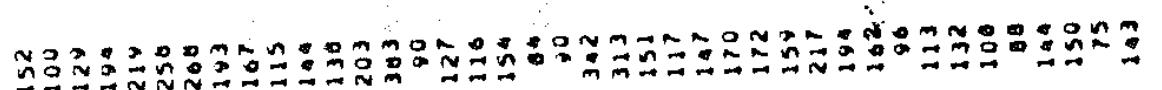

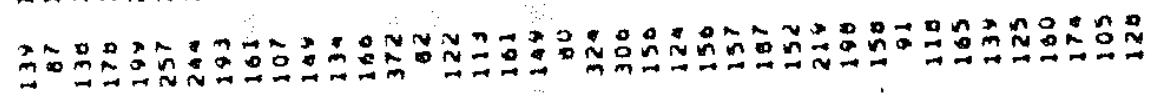

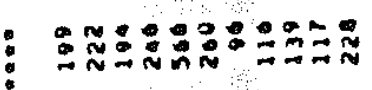
-

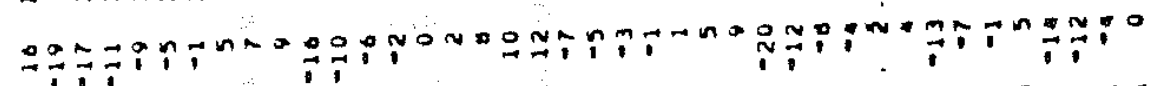

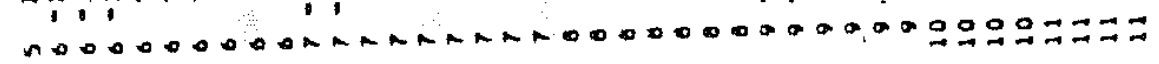

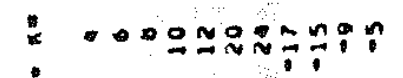
$0000000-\cdots-\infty$

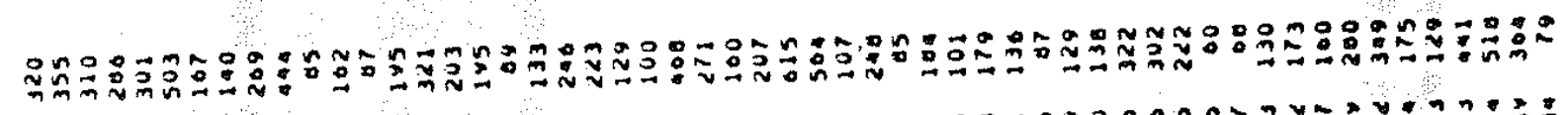

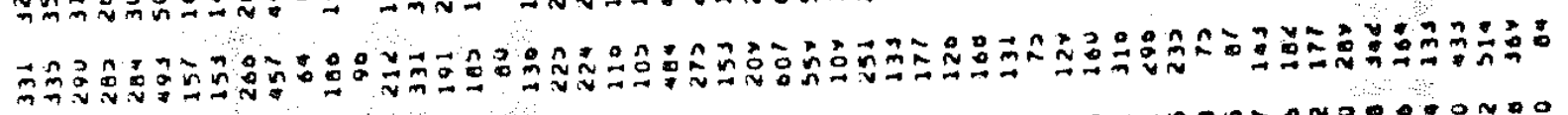

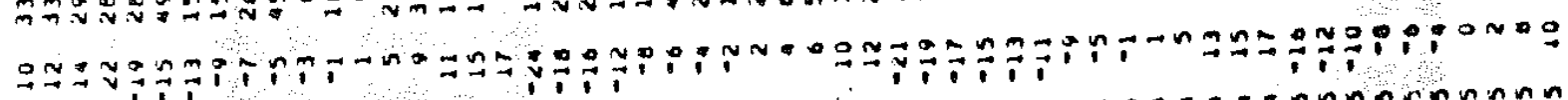

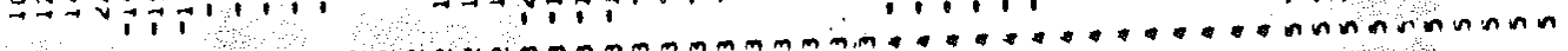

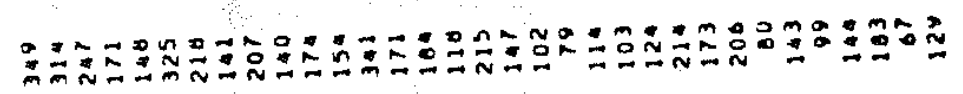

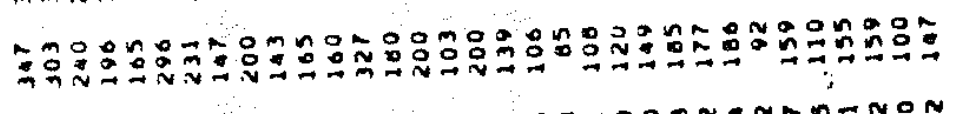
: $\dot{s}$

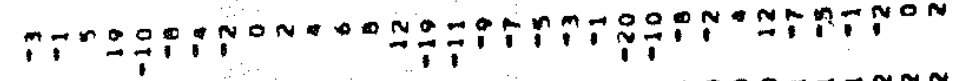

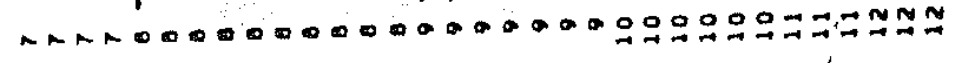

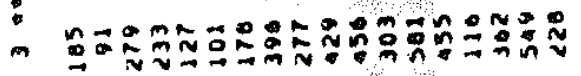
* unamanomoginonaco

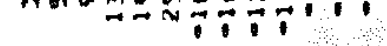

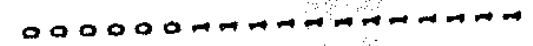

\section{(1)}

$$
\text { में }
$$

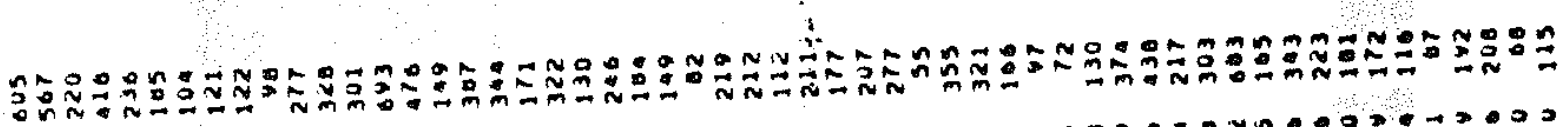

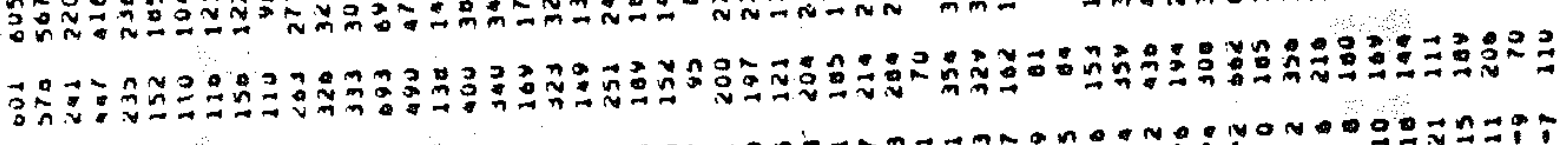
mMN $=$ =

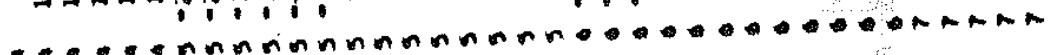

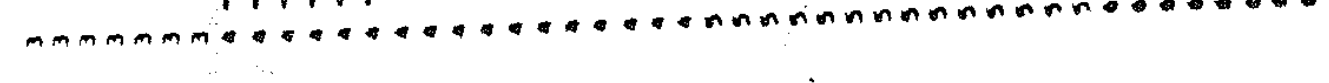




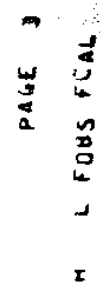

$\dot{\&} \cdot \cdot$

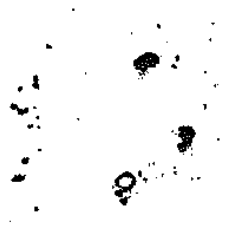

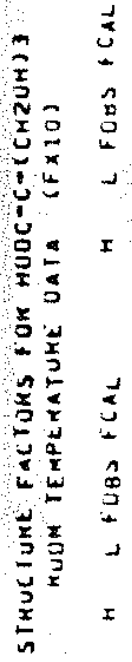

$\infty$

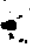
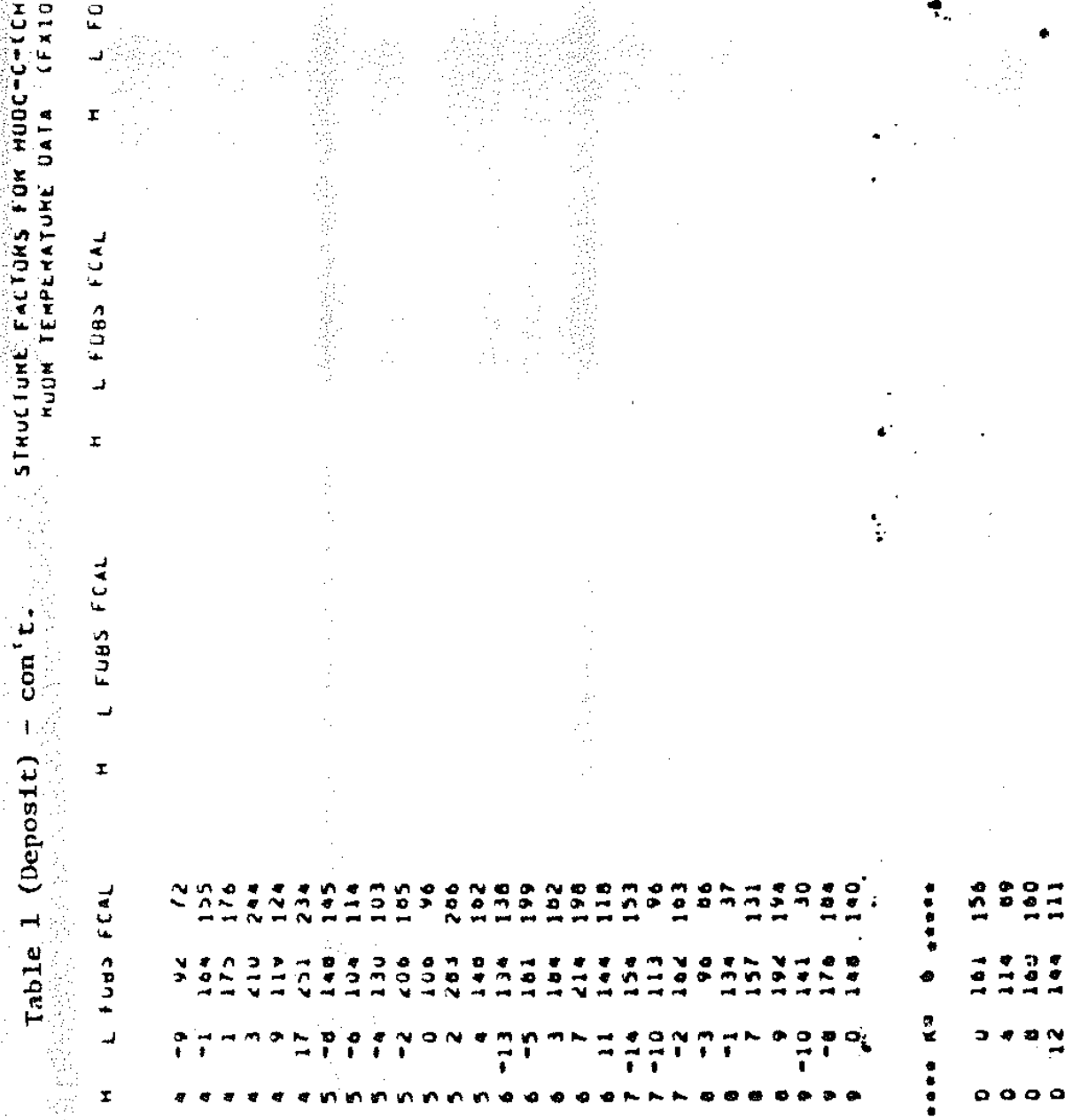

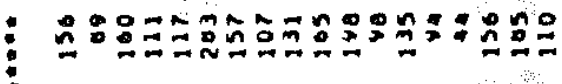

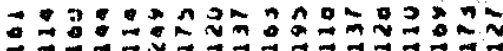


Table 3 (Deposit) Intramolecular bond angles in

tris (hydroxymethyl! acetic acid.

$$
\begin{aligned}
& \text { Atoms } \\
& C(4)-C(C)-C(2) \\
& C(4)-C(C)-C(3) \\
& C(4)-C(C)-C(1) \\
& C(2)-C(C)-C(3) \\
& C(2)-C(C)-C(1) \\
& C(3)-C(C)-C(1) \\
& \text { Average } C-C(C)-C
\end{aligned}
$$$$
O(1)-C(1)-C(C)
$$$$
0(2)-C(2)-C(C)
$$$$
O(3)-C(3)-C(C)
$$$$
\text { Averace } O-C-C(C)
$$$$
O(41)-C(4)-C(C)
$$$$
O(42)-C(4)-C(C)
$$$$
O(41)-C(4)-O(42)
$$$$
\text { Average } \mathrm{O}-\mathrm{C}-\mathrm{C}(\mathrm{C})
$$$$
H 11-C(1)-H 12
$$$$
\mathrm{H} 2 \mathrm{I}-\mathrm{C}(2)-\mathrm{E} 22
$$$$
431-C(3)-433
$$$$
\text { Averace } \mathrm{H}-\mathrm{C}-\mathrm{H}
$$

$$
\text { HII-C (I) }-O(1)
$$$$
\text { HI } 2-C(1)-O(1)
$$$$
\mathrm{H} 21-\mathrm{C}(2)-\mathrm{O}(2)
$$$$
122-C(2)-O(2)
$$$$
\mathrm{E} 31-\mathrm{C}(3)-O(3)
$$$$
132-C(3)-0(3)
$$$$
\text { Average } \mathrm{H}-\mathrm{C}-\mathrm{C}
$$

$111-C(1)-C(C)$

$$
\text { HI }-C(1)-C(C)
$$$$
1121-C(2)-C(C)
$$$$
\text { H:22-C(2) }-C(C)
$$$$
\text { H31-C (3) }-C(C)
$$$$
\text { H } 32-C(3)-C(C)
$$$$
\text { Average } \mathrm{H}-\mathrm{C}-\mathrm{C}(\mathrm{C})
$$

HOI-O(1) $-\mathrm{C}(1)$

$\mathrm{HO} 2-\mathrm{O}(2)-\mathrm{C}(2)$

HO3-O (3) $-\mathrm{C}(3)$

HO41-O (41)-C (4) Average $\mathrm{H}-\mathrm{O}-\mathrm{C}$

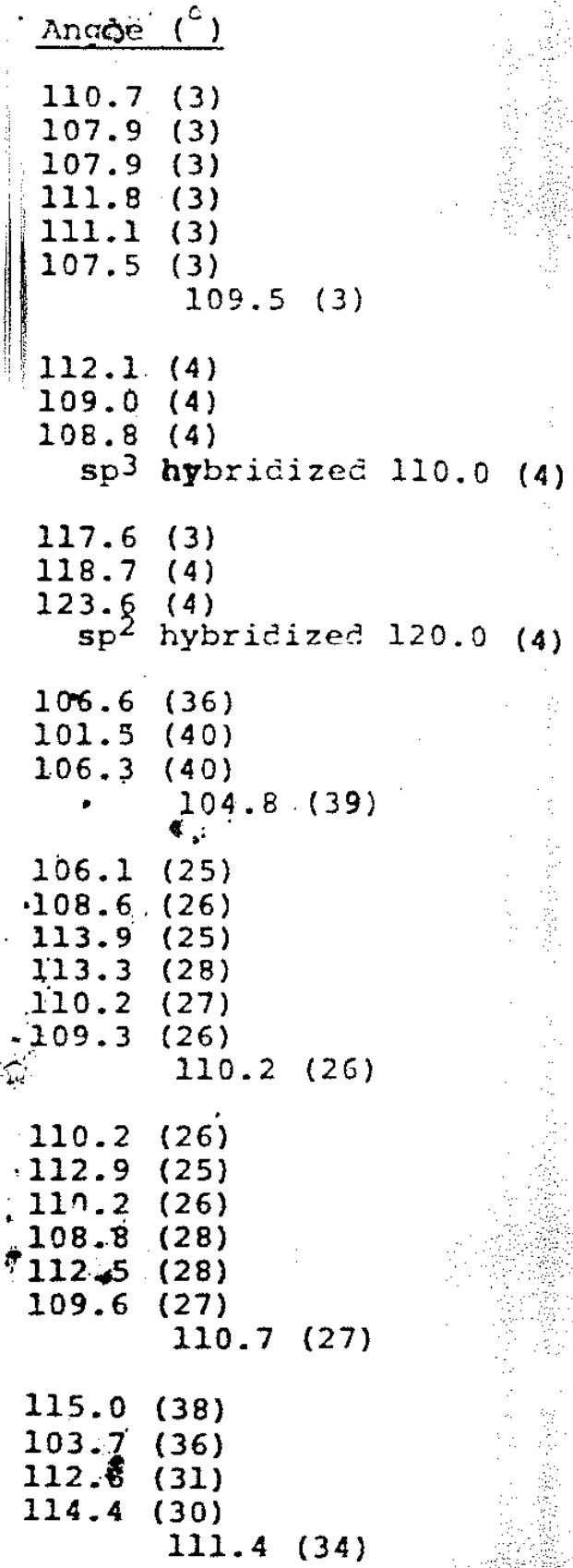


Fable 4 (Deposit) - TLS Tensors for Tris (hydroxymethyl)acetic Acid

RMS $\Delta U_{i j}\left(\AA^{2}\right)=0.0041$

L.S.D. $u_{i j}: 0.0050$

I. Tensor

$\left[\begin{array}{rrr}2.38 & -.24 & .28 \\ 2.51 & .17 \\ & 2.29\end{array}\right] \times 10^{-2} \AA^{2}$

L

$\left[\begin{array}{llr}18.498 & -2.696 & 1.075 \\ & 11.867 & -135 \\ 12.272\end{array}\right] \quad \mathrm{deg}^{2}$

5 Teisor - necligible

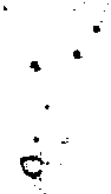

